of the appendix, 37 per cent.; and at the creal end of the appendix, 15 per cent. It will be seen that the terminal portion is affected in about half of the cases. A study of the records clemonstrates also that it has been found as (1) a hard, small, whitish nodule; (2) a more or less caseous little mass; (3) a nodule breaking down in the centre; (4) an apparently fibrous stricture; (5) an apparent obliteration of the lumen of the tube; and (6) a nodular bulbous tip to the organ. It would be folly to attempt to say which is the most common but it seems probable that if the strictures or local obliterations so often seen in the distal segment of the appendix were examined microscopically the forms of carcinoma associated with these conditions would be found to be the most frequent.

For a long time it has been known that the symptoms produced by a carcinoma are dependent almost entirely upon the anatomical or mechanical evils which its presence entails. For instance, the symptoms of benign and malignant disease of the pylorus are both due to the mechanical obstruction so cansed. With regard to the appendix, it is important that this should be taken into account. For the material and organisms imprisoned behind the carcinoma are very likely to precipitate an attack of appendicitis, and the offending organ sloughe, forming an abscess or killing the patient with peritonitis, or it is removed before dissemination of the growth has taken place. As a result we know very little indeed of the prognosis of these cases as to length of life. In almost every instance the report has been made about six months or a year after the operation, the patient being at the time well. In one case recorded by Lejars the patient died after a rapid return and dissemination of the growth. Otherwise the prospect of cure seems to be good, a fact which is surprising when one considers that most of the subjects are extraordinarily young and malignant disease at such ages spreads very rapidly. For instance, "the youngest (subject) was 12, the next 15, two at 19 , one at 20 , one at 23 , three at 24 , one at 25,26 , and 27 respectively, and two at 30 "-w that is to say, more than a third of them are under 30 years of age! The explanation given-namely, that either the growth sloughs or the patient dies from appendicitis or the organ is removed before dissemination has occurred-..-may be the correct one. In the example we report over three years have elap-ed and the patient when seen three months ago was still alive and well.

The patient, a woman, aged 31 years, unmarried, was admitted into St. Thomas's Hospital on Oct. 1st, 1901 She had suffered for some time from uterine trouble, for which four years ago she had been curetted. Six months previously to her admission into St. Thomas's Hospital she had been operated upon for hæmorrhoids. Two months later she had a second attack of pain in the right side which lasted two hours and caused her to faint. After a fortnight's interval she had a similar but less severe attack. The pain was over the right iliac fossa, low down. "On deep palpation in the right iliac region the patient complained of a little tenderness at a point midway between the umbilicus and the anterior superior spine of the ilium." The right kidney was found to be moveable. Per vaginam there was a hard mass to the right of the uterus depressing the vaginal fornix. This was thought to be either a solid tumour of the ovary (? malignant) or a subperitoneal fibroid burrowing into the broad ligament. The mass was equal in size to a pigeon's egg. The size of the aterus was normal. Operation was performed on Oct. 13th, 1901. The abdomen was opened in the middle line below the umbilicus. The tumour was found to be a small fibromyoma of the right broad ligament, close to, but not directly connected with, the uterus. After this had been enucleated the appendix was examined. It was brought into the wound and found to have only a few omental adhesions and to be apparently healthy, except that at the extreme tip it was thickened and bulbous. It was on this account removed. 'The specimen removed was one and a quarter inches in length. Its distal end was firm and bulbous. On opening. it the mucous membrane was seen to be somewhat thickened at its end and showed little injected points. The extreme tip was closed by a little nodule three-sixteenths by one-third of an inch in size which had the appearance of caseous material though somewhat harder. The lumen of the tube was quite abliterated at the tip but not elsewhere. 'The peritoneal coat arer the distal end was much thickened. Sections were cut and a report was made by Mr. S. G. Shattock and Dr. C. G. Seligmann, stating that the tumour was a spheroidal-celled

carcinoma. The wound healed by first intention. By Feb. 20th, 1902, the patient had gained nine pounds in weight and was looking much better. She has since presented herself periodically for examination. When last seen - namely, on June 18th, 1904-there was no evidence of mischief either in the pelvis or in the region of the appendix. She was very well except that she was still thin and had no appetite.

The above is thoroughly typical in every way of the kind of case in which carcinoma is found. Besides what has been said above, the subject was a young woman, and women are apparently more prone to be attacked than men, who only seem to be affected later in life. It is interesting to examine the symptoms in order to see which of them were due to the fibromyoma and which to the appendix. It seems, on the whole, that most of them were due to the former, though it is not easy to refer the sudden and severe pains in the right side to this cause. Perhaps the tumour may have caused some temporary obstruction to the right ureter. On the other hand, the nodule might be thought to have excited painful peristalsis (colic in the appendix), but as it affected the tip of the organ it would seem hardly able to do so. The tenderness over McBurney's point seems rather more likely to be due to the diseased appendix than to the fibromyoma. Finally, it may be pointed out that the concurrence of fibromyoma of the uterus and carcinoma of the appendix bas never been recorded before But Dr. Deaver in his "Treatise on Appendicitis," pp. 95, 96, reports a case of fibromyomata of the appendix, two in number, associated with similar tumours of the uterus. One was situated at the centre and the other towards the tip of the former. Both were spherical and about five millimetres in diameter. He also refers to a case of fibromyoma of the appendix, the tumour being single, which was not associated with any uterine disease.

\title{
INFLAMMATION OF THE MUSCLES, WITH SPECIAL REFERENCE TO TWO CASES OF INFECTIVE MYOSITIS.
}

By JOHN HILL ABRAM, M.D. LOND., M.R.C.P. LOND.,

PHYSICIAN TO THE LIVERPOOL ROYAL INFIRMARY; LECTURER ON TO THE LIVERPOOL ROYAL INFIRMARY; LECT
CLIMICAL MEDICINE, UNIVERSITY OF LIVERPOOL.

IT has been my fortune in the last two years to meet with two cases in which an inflammatory process in a muscle was a distinctive feature in the symptom-complex. In spite of their rarity muscle inflammations are important from their deadly character. Thus out of 17 cases of infective myositis alluded to by Lorenz ${ }^{1}$ seven were known to have died in from four to 16 days; in one of my cases death occurred on the sixth day. Again, in 15 cases of true dermatomyositis death followed in 11 and of five cases of polymyositis hæmorrhagica all died.

I have taken the subjoined classification from Lorenz's exhaustive monograph on "Muscle Diseases" :-

A. Suppurative varieties.

1. Traumatic.

2. By extension.

3. Metastatic.

4. Infective myositis.

B. Non-suppurative varieties.

1. Polymyositis. Dermatomyositis. P. hæmorrhagica.

P. with erythema multiforme.

I may add a few notes on the more unusual forms.

Dermatomyositis.- Not unlike trichinosis. Causation, unknown. After prodromal symptoms for several days marked fever, inflammation of the skin, subcutaneous tissue, and the muscles supervene. There is enlargement of the spleen as well as marked sweating. Death occurs from lesions of the respiratory muscles or the patient slowly recovers.

Polymyositis hemorrhagica.-Very similar to dermatomyositis but the heart is especially affected. 
Neuromyositis.-Closely related to multiple neuritis.

Myositis with joint lesions.-Acute rheumatism and gonorrhœea.

Myositis tuberculosa.-Fither hæmatogenous or dne to extension.

Myositis syphilitica.-Either a diffuse inflammation with skin implication or gumma.

Myositis fibrosa.-May occur as a definite disease, probably due to congenital tendency, but is more commonly a secondary condition.

Myositis ossificans.-(a) Local variety practically always traumatic in origin, and $(b)$ myositis ossificans progressiva affecting children and young adults, associated with inflammatory phenomena and probably due to congenital predisposition.

The most recent work has shown that micro-organisms play a large part in the immediate causation of muscle inflammation. In the suppurative varieties this has been definitely proved: streptococci, staphylococci, pneumococci, bacillus typhosus, and bacillus mallei have been demonstrated in particular cases. In the non-suppurative varieties, although this causation is probable, yet at present it is not supported by direct evidence. Dermatomyositis has been confused with trichinosis and this has led Unverricht to sugoest a gregarina invasion as a possible cause, such a condition being known in animals; this, however, has not yet been verified. Lessened resistance to infection from exposure to cold and exhaustion will naturally predispose to these lesions and the hæmorrhages known to occur in overworked muscles will afford a definite localising influence to excessive muscular action.

Brunon's list of muscles affected in infective myositis runs as follows: Pectoralis major, deltoid, triceps and biceps, psoas, quadriceps, and the gastrocnemius. In both of $m y$ cases the pectoralis major was the muscle affected. The inflammatory process attacks the interstitial tissue in the first instance and it is difficult to determine the part degeneration plass in the muscle-fibre changes. In acute cases fever is generally noted, a point of some importance in the diagnosis from muscular rheumatish. Attention will be drawn to the muscle or muscles by their becoming swollen and erentually hard and shortened when the whole belly thereof is involved; in other cases nodules may be found. The skin may or may not show œedema. When œedema is present the underlping muscle change is difficult to detect. In my first case it was not recognised until the second operation; in the second the odema was closely limited to the area of skin covering the muscle involved. Pain is a marked feature and is greatly increased on movement stretching the affected muscle; in myositis syphilitica it may only occur at night. Limitation of movement naturally is present, partly due to dread of pain but also to contracture induced by the irritation of sensory nerve fibres passing through the inflamed area.

I shall make special reference to infective myositis, which may be defined as a form of muscle inflammation not due to definite traumatism nor part of a general septico-pyæmia, yet running the course of an infective disease like osteomyelitis. Lorenz, writing in 1898, recognises but 17 cases up to that date. Miyake, ${ }^{2}$ writing in 1904, says that the disease is fairly common in Japan, 33 cases coming under his observation in 21 months. He gives, too, a better prognosis than usual, possibly due to the infection arising from staphylococci rather than streptococci.

In both of my cases strain was suggested as a predisposing cause. In the first case nothing further was made out, but in the second case Dr. H. E. Annett of the Liverpool Institute of Comparative Pathology, who kindly examined a portion of muscle for me, reports: "A smear of the pus show microscopically streptococci of two to eight members in the chain. Pus inoculated intraperitoneally into rabbit produced death in 36 hours-typical streptococcal infection."

The disease is met with in three distinct varieties, a malignant form killing in a few days, an acute form in which in spite of severe general symptoms recovery may occur, and a mild subacute form.

After the general description I have already given I need only record the details of my two cases.

CASE 1.-A male, aged 19 years, was admitted into my men's ward at the Royal Infirmary, Liverpool, on Nov. 10th, 1902. He stated that eight weeks before admission he thought he strained his arm at work for he was attacked

2 Mivake. Mittheiłungen aus dem Grenzgebieten der Medicin und Chirurgie, Band xiii., 2, 1904 . with severe pain in the chest. Three weeks later there was very marked limitation of movement and swelling over the upper left chest was noted; one week before admission a lump was noticed in the armpit. On admission he looked ill, the temperature was $102^{\circ} \mathrm{F}$, the pulse was 88 , regular and of poor tension, and the respirations were 20 . The left arm was kept close to the chest and passive movement was painful. A diffuse brawny swelling extended from the clavicle to the nipple on the left side. The skin of the anterior axillary fold was cracked and sore (the result of local applications). Just below the lower border of the pectoral muscle was an area of softening. An incision was made into the softened area on Nov. 21st and a further more extensive one on the 29th. Although the sheath of the pectoral muscle was freely opened healing. was slow. It was apparently hastened by the administration of protonuclein. The patient left the hospital on Dec. 30th practically well. CASE 2.-I saw the patient (a single woman, aged 45 years) on Sept. 4th, 1904, with Dr. Mary Birrell Davies. She was evidently deeply poisoned, the face was livid, and the expression was dull. The pupils were dilated. The temperature was $103^{\circ} \mathrm{F}$. and the pulse was 140 and very soft. The heart and lungs were normal. There was marked oedema of the skin over the right upper chest, limited very definitely below by the anterior fold of the axilla. A few scattered erythematous areas were also noted. No glands could be felt in the axilla. The shoulder-joint seemed to be intact. Mr. Douglas-Crawford cut down upon the pectoralis major later in the day and on opening the sheath of the pectoral muscle found suppuration in it but no definite abscess formation. The inflammatory process had extended along the suspensory ligaments of the breast. The patient succumbed a few hours later. Dr. Davies has very kindly written some notes bearing on the early history. The patient thought she had had a chill, as she was "shivery" on August 29th and 30th. On the 31st, when Dr. Davies first saw her, the temperature was $101^{\circ}$, the pulse was 110 and

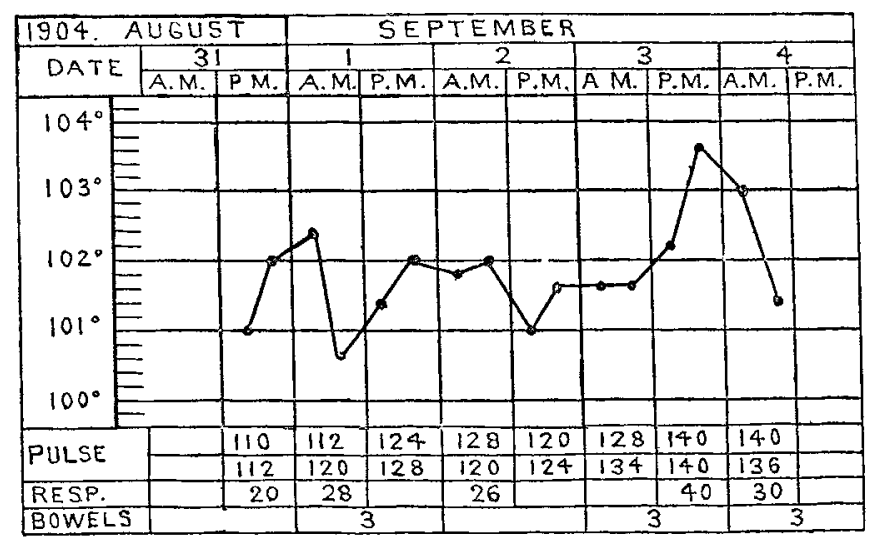

full, and there was pain in the right upper chest, the arm being kept closely to the side and movement being resisted. The first trace of odema over the muscle was noticed on Sept. 3rd and on the 4th death occurred, within a week from the initial shivering. (See chart appended.)

Finally, as regards treatment, general measures must be adopted to maintain the patient's strength; Dujardin Beaumetz ${ }^{3}$ speaks favourably of kola. I thought my first case was distinctly improved by the exhibition of protonuclein. I cannot say that in infective conditions I have noted much benefit from antiseptic drugs. Beaumetz speaks well of asaprol, a soluble naphthol, in daily doses of from 45 to 60 grains. Antistreptococcic serum should be usefu in streptococcus cases but at present the results therefrom in other streptococcus lesions are not altogether satisfactory. For my own part, I should urge speedy and free incision into the affected muscle even before definite abscess formation is perceptible.

In conclusion, I must express my thanks to Dr. Davies for the early notes of Case 2 and also for the temperature chart. Liverpool.

3 Dujardin Beaumetz: Twentieth Century Practice of Medicine.

Russian Rights to Dissect the Dead.-The infirmary medical officer (Dr. Putchkoff) of Moscow having applied to have the rights of the infirmary medical officer to dissect dead patients defined, has learned that such may not be allowed without the consent of the relatives except in casęs of certain specified crimes, 